\title{
Special issue on multi-terminal information theory
}

\author{
Andrew Thangaraj · Srikrishna Bhashyam
}

Published online: 26 March 2013

(C) Indian Institute of Technology Madras 2013

Information theory, founded by Shannon in the 1940s, has today grown to be an important part of communication system design. In a point-to-point channel, where a single transmitter attempts to communicate to a single receiver, the fundamental limits on communication rate predicted by information theory can be practically achieved by codes such as turbo and low-density parity-check codes. However, in several wireless communication systems, there are often scenarios where multiple transmitters send information to multiple receivers simultaneously. In such multiterminal scenarios, active research still continues on fundamental limits and coding schemes.

In this thematic volume, we have seven papers that bring out several facets of multi-terminal information theory in the current state of the art.

In the first paper titled "Physical layer network coding for wireless multi-way relaying with finite signal sets", multiway relaying where multiple nodes exchange information with the help of a relay node is considered. Recent results on physical layer network coding schemes for two-way and three-way relaying are reviewed and open problems in the multiway scenario are presented.

The second paper titled "Upper concave envelopes and auxiliary random variables" proposes a new characterization of inner and outer bounds of some network information theoretic regions and provides an alternative method for proving optimality of achievable rate regions.

\footnotetext{
A. Thangaraj · S. Bhashyam $(\bowtie)$

Department of Electrical Engineering,

IIT Madras, Chennai, India

e-mail: skrishna@ee.iitm.ac.in

A. Thangaraj

e-mail: andrew@ee.iitm.ac.in
}

The third paper titled "Capacity and fairness of the multiple access channel in energy harvesting wireless networks" considers a Gaussian multiple access channel with energy harvesting transmitters and develops adaptive transmission schemes in a flat fading scenario under a finite battery constraint.

The fourth paper titled "Optimum number of hops in linear multihop wireless networks" optimizes the number of hops to achieve maximum end-to-end throughput in a linear multihop relay network under both transmission and computation power constraints. The effect of interference cancellation and duplexing methods on throughput have been quantified.

The fifth paper titled "Multiuser cognitive radio networks: An information-theoretic perspective" derives achievable rate regions and outer bounds for three-user interference channels where the transmitters operate under some cognitive radio scenarios. Various message sharing scenarios and rate-splitting strategies relevant to the cognitive radio context are studied.

The sixth paper titled "Femtocell architectures with spectrum sharing for cellular radio networks" proposes femtocell network architectures for managing interference between femtocell and macrocell networks and achieves significant gains in sum rate.

The last paper titled "In-network estimation of frequency moments" considers distributed function computation using a randomized algorithm over a network and focusses on a class of large alphabet non-linear functions.

Finally, we would like to thank all the authors for their submissions and the reviewers for their careful review and constructive feedback. We would also like to thank the editorial and publishing team for their cooperation in bringing out this special issue. We hope that this collection of papers would be valuable to researchers in network information theory and related areas. 